\title{
Septic Shock in Pediatrics
}

\author{
Tarek Hamed Attia ${ }^{1}$, Maysaa Abdallah Saeed ${ }^{2}$ \\ ${ }^{1}$ Pediatric Department, Faculty of Medicine, Zagazig University, Egypt \\ ${ }^{2}$ Tropical Medicine Department, Faculty of Medicine, Zagazig University, Egypt
}

Corresponding Author Tarek Hamed Attia

Mobile:

$+201276099800$

E mail: tareqhamed@liv.com
Sepsis is defined as a clinical syndrome that complicates severe infection and is associated with the systemic inflammatory response syndrome as well as immune dysregulation. Circulatory decompensation and end-organ dysfunction will be the ultimate end if sepsis is not properly managed. In this syndrome, tissues remote from the original insult display the cardinal signs of inflammation, including

\section{DEFINITION}

Septic shock refers to sepsis with cardiovascular dysfunction that persists despite the administration of $\geq 40$ $\mathrm{mL} / \mathrm{kg}$ of isotonic fluid in one hour [1].

Pediatric sepsis is generally considered to comprise a spectrum of disorders that result from infection by bacteria, viruses, fungi, or parasites or the toxic products of these microorganisms. Early recognition and intervention clearly improve the outcome for infants and children with conditions that lead to sepsis [2].

The spectrum of sepsis ranges from microbial invasion of the bloodstream or intoxication with early signs of circulatory compromise including tachycardia, tachypnea, peripheral vasodilation, and fever (or hypothermia) to full-blown circulatory collapse with multiple organ dysfunction syndrome (MODS) and death [3].

How can we diagnose septic shock? A clinical diagnosis of septic shock is made in children who have signs of inadequate tissue perfusion, two or more criteria for the systemic inflammatory response syndrome, and suspected or proven infection [4]. Rapid recognition of hemodynamic abnormalities and early suspicion of infection are essential to achieve favorable outcomes. vasodilation, increased microvascular permeability and leukocyte accumulation. Recently pediatric mortality from severe sepsis and septic shock has markedly decreased because of early recognition, aggressive fluid therapy and early administration of vasoactive agents in addition to antibiotics.

\section{1- Signs of inadequate tissue perfusion}

Seriously ill patients should undergo urgent evaluation for the following signs of impaired perfusion or shock [5]:

- Fever.

- Tachycardia or bradycardia.

- Decreased peripheral pulses compared with central pulses.

- Mottled or cool extremities.

- "Flash" or $>3$ second capillary refill.

- Dry mucus membranes, sunken eyes, and decreased urine output.

- Tachypnea, bradypnea, or apnea

- Hypotension.

- Altered mental status (irritability, anxiety, confusion, lethargy, somnolence, apnea).

- Hypothermia (especially neonates.

\section{2- Signs of infection}

Patients should be simultaneously evaluated for an infectious source to distinguish non-infectious from septic shock. Fever, cough or congestion, dyspnea, hypoxemia, rash, abdominal pain, myalgias, immunocompromising condition (chemotherapy, sickle cell disease, or other known conditions associated with splenic dysfunction or primary immune deficiency), leukocytosis, or leukopenia with thrombocytopenia should raise suspicion for infection. Other factors concerning for specific infections, such as dysuria 
(urinary tract infection), hematochezia (gastroenteritis), headache and neck stiffness (meningitis), bone or joint inflammation (Staphylococcus aureus), conjunctival suffusion / injection (toxic shock syndrome), ecthyma (Pseudomonas species) and petechiae/purpura (meningococcemia), should also be quickly ascertained. If an infection is suspected or a non-infectious etiology of shock is not clear, current guidelines recommend obtaining blood, urine, and, as indicated, other cultures and administering empiric broad-spectrum antibiotics within one hour of presentation [6]. Antibiotic therapy should not be delayed beyond one hour in order to obtain cultures if there is a concern for severe sepsis or septic shock.

\section{3- Systemic inflammatory response syndrome}

The systemic inflammatory response syndrome (SIRS) is a widespread inflammatory response that may or may not be associated with infection. The presence of two or more of the following criteria (one of which must be abnormal temperature or leukocyte count) defines SIRS [3]:

- Core temperature (measured by rectal, bladder, oral, or central probe) of $>38.5^{\circ} \mathrm{C}$ or $<36^{\circ} \mathrm{C}$

- Tachycardia, defined as a mean heart rate more than two standard deviations above normal for age, or for children younger than one year of age, bradycardia defined as a mean heart rate $<10$ th percentile for age.

- Mean respiratory rate more than two standard deviations above normal for age or mechanical ventilation for an acute pulmonary process.

- Leukocyte count elevated or depressed for age, or $>10$ percent immature neutrophils

These original cut points that define age-specific SIRS criteria were chosen to be sensitive to physiological parameters indicative of a systemic inflammatory state, allowing for the maximum number of potential patients with sepsis to be identified for observational and clinical trials.

While several studies have demonstrated value and reproducibility of these SIRS criteria in the clinical trial setting [7], other vital sign ranges (such as those used in the Pediatric Advanced Life Support [PALS] septic shock guidelines [8] may be more informative to guide diagnostic and therapeutic decisions outside of the research setting and other cutoff values have been proposed for respiratory rates [9].

How can we investigate septic shock?

Suggested laboratory studies for children with sepsis and septic shock are [10]:
- Rapid blood glucose

- Arterial or venous blood gas

- Complete blood count with differential

- Blood lactate

- Serum electrolytes

- Blood urea nitrogen and serum creatinine

- Ionized blood calcium

- Serum total bilirubin and alanine aminotransferase

- Prothrombin and partial thromboplastin times (PT and PTT)

- International normalized ratio (INR)

- Fibrinogen and D-dimer

- Blood culture

- Urinalysis

- Urine culture

- Other cultures as indicated by clinical findings

- Diagnostic serologic testing as indicated to identify suspected sources of infection

- Inflammatory biomarkers (like, C-reactive protein, pro calcitonin) in selected cases

The following laboratory findings support the diagnosis of septic shock [11] :

- Lactic acidosis indicated by metabolic acidosis on blood gases and elevation of arterial blood lactate $(>3.5 \mathrm{mmol} / \mathrm{L})$

- Age-specific leukocytosis or leukopenia

- Platelet count $<80,000 /$ microL or a decline of 50 percent from highest value recorded over the past three days

- Disseminated intravascular coagulopathy (decreased fibrinogen with increased D-dimer, international normalized ratio, prothrombin time, or partial thromboplastin time)

- Renal insufficiency suggested by a serum creatinine $\geq 2$ times upper limit of normal for age or twofold increase in baseline creatinine.

- Liver dysfunction implied by a total bilirubin $\geq 4 \mathrm{mg} / \mathrm{dL}$ (not applicable to newborn) or alanine aminotransferase (ALT) $>2$ times upper limit of normal for age

- Pyuria indicating urinary tract infection

How can we monitor improvement of septic shock?

Restoration of tissue perfusion, such as reversal of shock, should be targeted to the following therapeutic endpoints (goals in parentheses) [6] :

- Quality of central and peripheral pulses (strong, distal pulses equal to central pulses)

- Skin perfusion (warm, with capillary refill $<2$ seconds)

- Mental status (normal mental status) 
- Urine output ( $\geq 1 \mathrm{~mL} / \mathrm{kg}$ per hour, up to $40 \mathrm{~mL}$ per hour, once effective circulating volume is restored)

- Blood pressure (systolic pressure at least fifth percentile for age: $60 \mathrm{mmHg}<1$ month of age, $70 \mathrm{mmHg}+[2 \mathrm{x}$ age in years $]$ in children 1 month to 10 years of age, $90 \mathrm{mmHg}$ in children 10 years of age or older); however, blood pressure by itself is not a reliable end point for assessing the adequacy of resuscitation

- Lactate $(<4 \mathrm{mmol} / \mathrm{L}$ or $\geq 10$ percent decrease per hour until normal)

- Central venous oxygen saturation $\left(\mathrm{ScvO}_{2}\right)$, ( $\geq 70$ percent), if available

\section{Management}

Goal-directed therapy for septic shock refers to an aggressive systematic approach to resuscitation targeted to improvements in physiologic indicators of perfusion and vital organ function within the first six hours. Effective treatment of septic shock requires rapid correction of circulatory dysfunction with continuous monitoring and re-evaluation at frequent intervals and early administration of empiric broad-spectrum antimicrobial therapy [4].

Whenever possible, children requiring resuscitation for septic shock should receive ongoing management by a pediatric critical care specialist or pediatrician with similar expertise in a pediatric intensive care unit.

Prompt identification and treatment of the source of infection are essential to successful management of septic shock and constitute critical interventions that can reverse septic shock. In contrast, other therapies (fluid administration, vasoactive drug infusion, or mechanical ventilation) are purely supportive in nature [6].

Oxygenation should be monitored using continuous pulse oximetry ( $\mathrm{SpO} 2)$. Patients should continue to receive supplemental oxygen to maintain oxygen saturation at 100 percent (patients with continued shock) or 97 percent (patients with restored perfusion and blood pressure) [12].

The clinician should determine the need for invasive monitoring via intra-arterial and central venous cannulas [13].

The need for aggressive administration of fluids to optimize tissue perfusion and to achieve physiologic goals typically continues beyond the first hour of care in children with septic shock. In patients with persistent poor perfusion or hypotension, boluses of fluids should continue until the central venous pressure is 8 to 12 $\mathrm{cmH}_{2} \mathrm{O}$ (12 to $15 \mathrm{cmH}_{2} \mathrm{O}$ in mechanically ventilated patients) or evidence of cardiac insufficiency (pulmonary edema, enlarged heart) occurs. The volume per bolus and types of fluid are as for the resuscitation period. Fluid input and output should be carefully monitored on an hourly basis [6]

Hemoglobin is the primary determinant of blood oxygen carrying capacity and, therefore, of tissue oxygen delivery. Thus, maintaining adequate hemoglobin levels is a critical aspect of managing children with septic shock. We suggest a hemoglobin goal of $10 \mathrm{~g} / \mathrm{dL}$ (equivalent to 30 percent hematocrit) as a target to be maintained with blood transfusion during resuscitation and ongoing management of children with septic shock [14].

\section{Disseminated intravascular coagulation and septic shock}

Patients with septic shock frequently have disseminated intravascular coagulopathy that may warrant treatment. Thus, baseline measures of clotting status should be routinely obtained in children with septic shock [2].

The goal of replacement therapy is to reduce or stop significant bleeding. Although replacement therapy should not be used to normalize laboratory tests (which often is impossible), a reasonable guide for the judicious use of blood components in the setting of significant bleeding includes maintaining platelet counts $>50,000$ per $\mathrm{mm}^{3}$ and fibrinogen concentration $>100 \mathrm{mg} /$ $\mathrm{dL}(1 \mathrm{~mol} / \mathrm{L})$.

Clotting factors can be replaced by either FFP or cryoprecipitate. FFP provides both procoagulant and anticoagulant proteins and is administered every 12 to 24 hours at a dose of 10 to $15 \mathrm{~mL} / \mathrm{kg}$ per infusion. Cryoprecipitate has higher concentrations of factor VIII and fibrinogen, and can be used to correct hypofibrinogenemia. It is administered every six hours as needed at a dose of $10 \mathrm{~mL} / \mathrm{kg}$ per infusion. Platelet transfusions are administered with a goal of maintaining the platelet counts $>50,000$ per $\mathrm{mm}^{3}[\mathbf{1 5}]$.

\section{Correction of metabolic derangement associated with septic shock}

Hypoglycemia remains a concern during the initial management phase of septic shock. Children have limited glycogen stores and may develop profound hypoglycemia during periods of stress. Thus, blood glucose should be monitored frequently upon admission and at least 
every six hours while the patient is unstable and corrected.

Hyperglycemia is commonly present in children with septic shock. insulin can be used to maintain euglycemia in these patients [16].

Adequate calcium stores are essential for maintaining myocardial contractility. Thus, ionized blood calcium levels should be monitored every one to two hours during initial management of septic shock. Patients with persistent shock and an ionized calcium $<1.1 \mathrm{mmol} / \mathrm{L}(4.8 \mathrm{mg} / \mathrm{dL})$ or those with symptomatic hypocalcemia (eg, positive Chvostek or Trousseau signs, seizures, prolonged QT interval on EKG, or cardiac arrhythmias) in association with a an ionized calcium <1.1 mmol/L ( $4.8 \mathrm{mg} / \mathrm{dL})$ should undergo correction with calcium gluconate 10 percent solution in a dose of $50 \mathrm{mg} / \mathrm{kg}(0.5 \mathrm{~mL} / \mathrm{kg})$, maximum dose $2 \mathrm{~g} \mathrm{(}(20 \mathrm{~mL})$ by slow intravenous or intraosseous infusion over five minutes. This suggested dose is equivalent to elemental calcium $5 \mathrm{mg} / \mathrm{kg}(0.15 \mathrm{mmol} / \mathrm{kg})$, up to $180 \mathrm{mg}$ elemental (4.5 mmol) per single dose [17].

Patients with septic shock who are receiving replacement therapy for adrenal insufficiency should receive stress doses of corticosteroids.

Similarly, children with septic shock and hypothyroidism should continue to receive thyroid replacement with levothyroxine [18].

\section{REFRACTORY SEPTIC SHOCK}

Fluid-refractory, catecholamine-resistant shock is defined as cardiovascular dysfunction despite at least $60 \mathrm{~mL} / \mathrm{kg}$ of fluid resuscitation and dopamine $\geq 10 \mathrm{mcg} / \mathrm{kg} / \mathrm{min}$ and/or direct-acting catecholamine (epinephrine, norepinephrine). Principles of management for children with refractory septic shock include treatment of reversible etiologies, stress dose corticosteroid therapy for patients with absolute adrenal insufficiency, and combination vasoactive drug therapy targeted to maintaining central venous oxygen saturation $\geq 70$ percent and normalizing blood lactate levels.

Although cardiac index targets are mentioned in previous pediatric septic shock guidelines, evidence of improved outcomes from routine measurement of cardiac index is lacking. If performed, the target range is 3.3 to 6.0 $\mathrm{L} / \mathrm{min} / \mathrm{m} 2$ [19].

\section{Correction of reversible conditions}

Pneumothorax, pericardial tamponade, and intraabdominal complications (like, peritonitis or ascites) comprise mechanical causes of shock that can be reversed by chest tube thoracotomy, pericardiocentesis, or abdominal decompression surgery, respectively. Drainage or debridement of infection sites (like, necrotizing fasciitis) or broadening of antimicrobial coverage are additional actions that may be warranted [20].

\section{Is there a rule for extracorporeal membrane oxygenation (ECMO)?}

American College of Critical Care Medicine (ACCM) suggest that children with persistent catecholamine-resistant shock in whom physiologic targets cannot be attained with fluid repletion, vasoactive infusion, and hormonal therapy; who do not have an immediately reversible cause, such as myocarditis, pneumothorax, or pericardial effusion; and who have a high likelihood of mortality, be evaluated for extracorporeal membrane oxygenation (ECMO) support, if available. If ECMO is not available at the facility in which the child is receiving care then the potential benefits of ECMO must be weighed against the likelihood that the patient can tolerate transfer [21].

What is place of Intravenous immune globulin in management of septic shock?

Adjuvant therapy with intravenous immune globulin (IVIG) has been proposed but evidence for benefit in children with septic shock remains inconclusive. A trial of polyclonal IVIG in 100 children with pediatric sepsis syndrome showed a significant reduction in mortality (28 versus 44 percent), length of stay (six versus nine days), and less progression to complications (8 versus 32 percent) [22].

Is there a rule of Plasma exchange or plasmapheresis in the future?

There has been considerable interest in extracorporeal filtration of circulating inflammatory mediators in sepsis. Although multiple studies in adults have been published on plasma exchange and plasmapheresis in sepsis, most are limited by small sample size at single institutions with considerable variability in the protocols utilized. Thus, current evidence is conflicting as to clinical benefit [23].

\section{PROGNOSIS}

Early recognition and management of pediatric severe sepsis and septic shock can be improved 
through the establishment of institutional care guidelines.

Many Factors are affecting the prognosis of septic shock. The most important are related to the host. Case fatality rates in children with severe sepsis are highest for infants 1 to 12 months of age (approximately 11 percent) and are higher across all age groups for children with comorbidities, especially in children with cancer or human immunodeficiency virus infection (12 to 16 percent) [24]. Site of infections is another prognostic factor. Children with endocarditis, central nervous system infection, and primary bacteremia have high case fatality rates (15 to 20 percent) [3]. The case fatality rate is lowest for genitourinary tract infections (approximately 4 percent) [25]. Microorganism may influence the progression from systemic inflammatory response syndrome to severe sepsis or septic shock and provide predictors of mortality. Case fatality is increased in children with pneumococcal and fungal infections (15 and 13 percent, respectively) [3]. Infection with organisms resistant to antibiotics (like, methicillin-resistant Staphylococcus aureus or vancomycin-resistant enterococcus species) is associated with a marked increased mortality from sepsis [26]. Mortality increases markedly depending upon the severity of illness in children with sepsis [27]. The development of multiple organ dysfunction indicates an increased severity of illness in patients with sepsis and is associated with a higher mortality estimated as 0 to 7 percent for patients with one affected organ system and 20 to 50 percent with two or more failing organ systems [24]. Treatment requirements are also important prognostic indicators. The need for multiple vasoactive infusions predicts a poor prognosis [28].

\section{REFERENCES}

1. Hotchkiss RS, Karl IE. The pathophysiology and treatment of sepsis. N Engl J Med 2003; 348:138.

2. Poggi C, Bianconi T, Gozzini E, Generoso M, Dani C. Presepsin for the Detection of Late-Onset Sepsis in Preterm Newborns. Pediatrics. 2014 Dec 15. [Medline].

3. Goldstein B, Giroir B, Randolph A. International pediatric sepsis consensus conference: definitions for sepsis and organ dysfunction in pediatrics. Pediatr Crit Care Med. 2005 Jan. 6(1):2-8.
4. Juskewitch JE, Prasad S, Salas CF, Huskins WC. Reliability of the identification of the systemic inflammatory response syndrome in critically ill infants and children. Pediatr Crit Care Med 2012; 13:e55

5. Brierley J, Carcillo JA, Choong K, Cornell $\mathrm{T}$, Decaen A, Deymann A, et al. Clinical practice parameters for hemodynamic support of pediatric and neonatal septic shock: 2007 update from the American College of Critical Care Medicine. Crit Care Med 2009; 37:666.

6. Kleinman ME, de Caen AR, Chameides L, Atkins DL, Berg RA, Berg MD, et al. Pediatric basic and advanced life support: 2010 International Consensus on Cardiopulmonary Resuscitation and Emergency Cardiovascular Care Science with Treatment Recommendations. Pediatrics 2010; 126:e1261.

7. Paul R, Melendez E, Stack A, Capraro A, Monuteaux M, Neuman MI. Improving adherence to PALS septic shock guidelines. Pediatrics 2014; 133:e1358.

8. Nakagawa S, Shime N. Respiratory rate criteria for pediatric systematic inflammatory response syndrome. Pediatr Crit Care Med 2014; 15:182.

9. Levy MM, Fink MP, Marshall JC, Abraham E, Angus D, Cook D, et al. 2001 SCCM/ESICM/ACCP/ATS/SIS International Sepsis Definitions Conference. Intensive Care Med 2003; 29:530.

10. Dellinger RP, Levy MM, Rhodes A, Annane D, Gerlach H, Opal SM, et al. Surviving sepsis campaign: international guidelines for management of severe sepsis and septic shock: 2012. Crit Care Med 2013; 41:580.

11. Dugas MA, Proulx F, de Jaeger A, Lacroix J, Lambert M. Markers of tissue hypoperfusion in pediatric septic shock. Intensive Care Med 2000; 26:75.

12. Brun-Buisson C, Doyon F, Carlet J, Dellamonica $\mathrm{P}$, Gouin F, Lepoutre A, et al. Incidence, risk factors, and outcome of severe sepsis and septic shock in adults. A multicenter prospective study in intensive care units. French ICU Group for Severe Sepsis. JAMA 1995; 274:968.

13. Velissaris D, Pierrakos C, Scolletta S, De Backer $\mathrm{D}$, Vincent JL. High mixed venous oxygen saturation levels do not exclude fluid responsiveness in critically ill septic patients. Crit Care 2011; 15:R177.

14. Marshall JC, Reinhart K, International Sepsis Forum. Biomarkers of sepsis. Crit Care Med 2009; 37:2290. 
15. Myburgh J, Finfer S. Causes of death after fluid bolus resuscitation: new insights from FEAST. BMC Med 2013; 11:67.

16. Maat M, Buysse CM, Emonts M, Spanjaard L, Joosten KF, de Groot R, et al. Improved survival of children with sepsis and purpura: effects of age, gender, and era. Crit Care 2007; 11:R112.

17. Branco RG, Garcia PC, Piva JP, Casartelli $\mathrm{CH}$, Seibel V, Tasker RC. Glucose level and risk of mortality in pediatric septic shock. Pediatr Crit Care Med 2005; 6:470.

18. Zaloga GP, Chernow B. The multifactorial basis for hypocalcemia during sepsis. Studies of the parathyroid hormone-vitamin D axis. Ann Intern Med 1987; 107:36.

19. Menon K, Ward RE, Lawson ML, Gaboury I, Hutchison JS, Hébert PC, et al. A prospective multicenter study of adrenal function in critically ill children. Am J Respir Crit Care Med 2010; 182:246.

20. Melendez E, Bachur R. Advances in the emergency management of pediatric sepsis. Curr Opin Pediatr 2006; 18:245.

21. Larsen GY, Mecham N, Greenberg R. An emergency department septic shock protocol and care guideline for children initiated at triage. Pediatrics 2011; 127:e1585.

22. MacLaren G, Butt W, Best D, Donath S. Central extracorporeal membrane oxygenation for refractory pediatric septic shock. Pediatr Crit Care Med 2011; 12:133.
23. El-Nawawy A, El-Kinany H, Hamdy El-Sayed $\mathrm{M}$, Boshra N. Intravenous polyclonal immunoglobulin administration to sepsis syndrome patients: a prospective study in a pediatric intensive care unit. J Trop Pediatr 2005; 51:271.

24. Nguyen TC, Han YY, Kiss JE, Hall MW, Hassett $\mathrm{AC}, \mathrm{Jaffe} \mathrm{R}$, et al. Intensive plasma exchange increases a disintegrin and metalloprotease with thrombospondin motifs-13 activity and reverses organ dysfunction in children with thrombocytopenia-associated multiple organ failure. Crit Care Med 2008; 36:2878.

25. Kutko $\mathrm{MC}^{1}$, Calarco MP, Flaherty MB, Helmrich RF, Ushay HM, Pon S,et al. Mortality rates in pediatric septic shock with and without multiple organ system failure. Pediatr Crit Care Med 2003; 4:333.

26. Watson RS, Carcillo JA, Linde-Zwirble WT, Clermont G, Lidicker J, Angus DC. The epidemiology of severe sepsis in children in the United States. Am J Respir Crit Care Med 2003; 167:695.

27. Jaramillo-Bustamante JC, Marín-Agudelo A, Fernández-Laverde M, Bareño-Silva J. Epidemiology of sepsis in pediatric intensive care units: first Colombian multicenter study. Pediatr Crit Care Med 2012; 13:501.

28. Proulx F, Fayon M, Farrell CA, Lacroix J, Gauthier M.Epidemiology of sepsis and multiple organ dysfunction syndrome in children. Chest 1996; 109:1033. 